Disclosure: No significant relationships.

Keywords: multidimensional evaluations; psychometric evaluation; Intellectual disability

\section{Mental health care}

\section{3}

Post traumatic stress disorder symptoms and stress burden among caregivers of patients with severe mental illness

\author{
A. Rady ${ }^{\star}$, T. Mouloukheya and E. Ramadan \\ Psychiatry, Alexandria University School of Medicine, Alexandria, \\ Egypt \\ ${ }^{\star}$ Corresponding author. \\ doi: 10.1192/j.eurpsy.2021.353
}

Introduction: Care givers of patients with severe mental disorders have been shown to be under heavy stress burden that reflect itself through various heterogenous psychiatric symptoms that may mimic PTSD with associated negative impact on interpersonal relations and work performance

Objectives: to assess the prevalence of PTSD symptoms among care givers of patients with severe mental illness

Methods: 70 patients care givers of sevely mentally ill patients compred to control 70 care giver of patients with chronic debilitating medical illness were recruited from outpatient of the university hospital outpatient facilities, random selection. Severe mental illness was defined by Global assessment of function GAF score above 50 and duration exceeding 2 years. Both groups were subject to Zarit burden interview to assess stress burden and post traumatic stress diagnostic scale PDS to assess PTSD symptomats

Results: $43 \%$ of care givers of severly mentally patients showed moderate to severe burden on the Zarit scale compared to only $10 \%$ among care givers of medically ill patients, this difference was statistically significant $(\mathrm{p}<0,001)$. Among care givers of severly mental patients showed moderate to severe score on post traumatic stress diagnostic scale compared to $0 \%$ among those taking care of medically ill patients. this difference was statistically significant $(p<0,001)$ Conclusions: Stress burden among care givers of patients with severe mental illness is high and may manifest symptoms of post traumatic disorder. This highlight the importance of particular psychological support and assessment among care givers of patients with sever mental illness

Disclosure: No significant relationships.

Keywords: ptsd; stress burden; Mental illness; care giver

\section{4}

Utilizing a novel digital affect mirror, morphii, to assess affective determinants of health

M. Milanak ${ }^{1 \star}$ and B. Sullivan ${ }^{2}$

${ }^{1}$ Psychiatry \& Behavioral Sciences, Medical University of South Carolina (MUSC), Charleston, United States of America and

${ }^{2}$ Research \& Development, ADoH Scientific, Mt. Pleasant, United States of America
${ }^{*}$ Corresponding author.

doi: 10.1192/j.eurpsy.2021.354

Introduction: Decades of research have shown that affect, emotions and moods, significantly impact all aspects of health behaviors. This research utilized a novel digital analogue technology (Morphii) to assess eight affective domains: stress, anxiety, loneliness, irritability, depression, pain, energy and overall feelings of wellness.

Objectives: To demonstrate the feasibility of use and strength of relationship/comparison to validated measures.

Methods: A U.S. census-based sample of adults ages 18-80 ( $\mathrm{n}=985)$ completed online assessments including the 8 Morphii's and additional comparative mental/behavioral health assessments (PSS-4, GAD-7, UCLA Loneliness Scale V3, BITe, PHQ-8 \& PHQ-2, P4 Pain Scale, WHO-5, CFQ-11, ESS, and Vitality Subscale SF-36) via the Prolific Academic online platform and were compensated nominally for their participation. The resulting sample was $51.6 \%$ female and $74.2 \%$ White.

Results: Each Morphii was compared with the common corresponding industry assessment (e.g., Depression Morphii with PHQ) resulting in Pearson correlations ranging from -.519 to .761 , with 6 of the 8 showing correlations above .700 . Pearson correlations between dysfunction and each of the 8 Morphiis were significant at the $\mathrm{p}<.000$ level, ranging from a low of .421 (Loneliness) to a high of .607 (Depression). Internal reliability was very good (Cronbach's Alpha $=.862$ ). Respondents who expressed an assessment modality preference (55.2\%) chose the Morphii type over traditional assessment format at a 2.5:1 ratio.

Conclusions: Morphii provides a reliable and valid assessment option with the ability to obtain a comprehensive (8 domains at once), efficient (less than 60 second administration), assessment with increased patient/client preference and engagement.

Disclosure: Milanak - submitting author - I serve on the advisory board for ADoH Scientific to consult on scientific research of Morphii development. To date, I have not been paid any money for this advisory role.

Keywords: Assessment; Affect; healthcare analytics; Whole person health

\section{5}

Experiences of fear in hospital settings from the perspectives of mental health service users and informal caregivers

T. Lantta ${ }^{1}$, M. Anttila ${ }^{1}$, J. Varpula $^{1}$ and M. Välimäki ${ }^{1,2}$

${ }^{1}$ Department Of Nursing Science, University of Turku, UNIVERSITY OF TURKU, Finland and ${ }^{2}$ Xiangya School Of Nursing, Central South University, Changsha, China

${ }^{*}$ Corresponding author.

doi: 10.1192/j.eurpsy.2021.355

Introduction: In the literature, service users and informal caregivers have been critical towards psychiatric inpatient care. However, little is known about their fears related to hospital care.

Objectives: We describe service users' and informal caregivers' experiences of fear in psychiatric hospital settings.

Methods: The data were collected from seven mental health associations located in six Finnish cities. Focus group interviews $(\mathrm{f}=8)$ were conducted (2015-2016) with service users $(n=20)$ and 